\title{
Fluidization Characteristics of Nano Particles with the Assist of Stirrer
}

\author{
Asadollah Kariman ${ }^{1}$, V. V. Basava Rao ${ }^{2}$, Majid Farjpourlar ${ }^{3}$ \\ 1,2,3. Department of Chemical Engineering, University College of Technology, Osmania University, \\ Hyderabad-07, India
}

\begin{abstract}
Fluidizationof ultrafine solidparticles iswidelyusedin a varietyofindustrial applicationsbecauseofitsunusualcapabilityofcontinuouspowderhandling, good mixing,large gassolidcontactarea, andveryhigh ratesofheatandmass transfer.As theparticlesizedecreases thecohesive forces betweenparticlesincreases.Therefore,fluidization of ultrafineparticles becomes much moredifficultas compared to thelargersizeparticlesanditneeds efficient method.

In thepresentwork, the fluidizationexperimentswereconducted usingairas fluidizingmediaand $\mathrm{SiO}$, $\mathrm{ZnO}$ and Al2O3 nano particles, with a size ranging from 15 to $30 \mathrm{nmin} 2$-dimensional fluidizedbedwiththe assist of stirrer.

The fluidization behavior of the nanoparticle, including the fluidization regime, the minimum fluidization velocity, and the bed expansion are also investigated. The results showed that smoother fluidization was observed with increasing agitation speed, because the agglomeration and channeling were reduced by the mechanical agitation. Itwas found that with the assist of stirrer, by increasing in the speed of stirrer initial bed voidage increased and terminal velocity and agglomerate diameter decreased.

Keywords: Fluidization, nanoparticles, bed expansion, stirring
\end{abstract}

\section{Introduction}

When a liquid or gas is passed at very low velocity up through a bed of solid particles, the particles do not move. If the fluid velocity is steadily increased, the pressure drop and the drag on individual particles increase and eventually the particles start to move and become suspended in the fluid, the terms fluidization and fluidized bed are used to describe the condition of fully suspended particles. Since the suspension behaves as a dense fluid, if the bed is tilted the top surface remains horizontal and the large objects will either float or sink in the bed depending on their density relative to their suspension. The fluidized solids can be drained from the bed through pipes and valves just as a liquid can and this fluidity is one of the main advantages of fluidization for handling of solids.

A fluidized bed is formed when a quantity of a solid particulate substance (usually present in a holding vessel) is placed under appropriate conditions to cause the solid/fluid mixture to behave as a fluid. This is usually achieved by the introduction of pressurized fluid through the particulate medium. This results in the medium then having many properties and characteristics of normal fluids; such as the ability to free-flow under gravity, or to be pumped using fluid type technologies. The resulting phenomenon is called fluidization.

Gas fluidization of powders has usually been restricted to Geldart (1973) group A and B powders. Finer particles in the group $\mathrm{C}$ range $(<30 \mu \mathrm{m})$ are difficult to fluidize because of the strong interparticle (cohesive) forces between them. A number of studies (Chaouki et al., 1985; Iwadate and Horio, 1998; Morooka et al., 1988; Pacek and Nienow, 1990; Wang, Y., et al., 2002; Wang, Z., et al., 1998; Zhou and Li, 1999) have been conducted to investigate the fluidization of ultrafine particles in conventional fluidized beds. Some of these investigators used nanoparticles, which are at the extreme end of Geldart group C powders. It has been observed that group C particles including nanoparticles can sometimes be fluidized in the form of ag- glomerates, and has been called "agglomerating fluidization" by Iwadate and Horio (1998). However, the formation of agglomerates and agglomerating fluidization occur at a super- ficial velocity well above the theoretical minimum fluidization velocity. The work reported in the literature thus far includes experiments and theoretical modeling of hydrodynamic behavior and agglomerate size.

\section{Experimental}

A schematic diagram of the gas fluidization system is shown in the figure 3-1. The system consists of a fluidized bed of fine particles, a flow meter and manometer to measure the pressure gradients. The fluidized bed is a vertical transparent column with a distributor at the bottom consisting of a ceramic plate about $2 \mathrm{~mm}$ thick with a pore size of $20 \mu \mathrm{m}$. The column is a glass tube with an inner diameter of $34 \mathrm{~mm}$ and an outer diameter of $50 \mathrm{~mm}$ and a height of about one meter. To generate a uniform gas field, glass beads are placed in a chamber placed below the distributor and above the gas inlet to form a packed bed about $50 \mathrm{~mm}$ high. This is tightly attached to the column with the help of gasket ,so that there is no leakage of air.

A wet filter is placed at the gas outlet to entrap any fine particle agglomerates. The filter may be dried later to obtain the fine particles back. During fluidization process a stirrer (a rod promoter) was hanged from the top of the fluidized column to vibrate the bed as shown in. The stirrer was connected to a motor and speed of rotation was varied by a Varriac. 


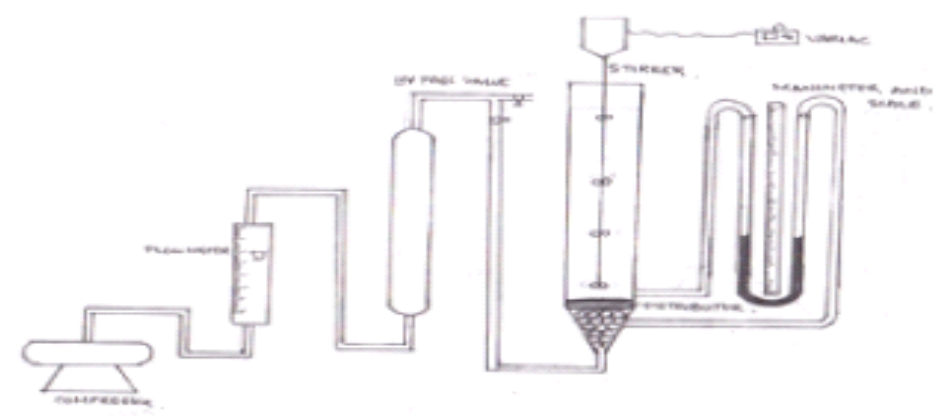

Figure 1 Schematic diagram experimental Set-up

The compressor is connected to one end of the flow meter and the other end of the flow meter is connected to the bottom of the chamber containing the glass beads. The glass beads help in generating a uniform gas field. On the top of the vertical glass column, an ultrafine mesh or a wet filter is placed. Pressure taps can be seen above and below the distributor, which is placed just above the chamber containing the glass beads, to measure the bed pressure drop. The pressure taps are connected to a $U$ tube manometer through which the bed pressure drop is calculated. Pressure taps are present at the middle and top of the column.

Before the experiment is begun, the sand particles are sieved through an appropriate mesh and their size and particle diameter is determined. Sieving also serves to remove any large agglomerates formed during packing, storage and transportation. However, because of fragmentation and re-agglomeration during fluidization, some agglomerates in the bed might exceed the sieve openings. Because of the surface treatment by the manufacturer, the fine particles may either be hydrophilic or hydrophobic. To minimize the effect of humidity on the fine particle fluidization, pure air from a compress ed air tank is used as the fluidizing gas.

The gas flow rate is measured and adjusted by using U-Tube manometer. The bed pressure drop is measured between the two pressure taps, one located at the top of the column near the flow exit and the other slightly above the distributor, so that it is not necessary to measure the pressure drop across the distributor. U tube manometers are used to measure pressure drops. Pressure taps also placed at elevated height on the column to measure the pressure gradients. A stirrer of one meter length along with stirring leaves placed at equal lengths is introduced into the glass column to ensure uniform mixing.

Particles are placed in the vertical column via opening at the top. Wet filter/ ultrafine mesh is fitted at the top and the particles are allowed to settle. The initial height of the bed is noted down. Compressed gas, set at a certain flow rate is allowed to move through the vertical column via the chamber of glass beads, for uniform distribution, and move thorough the pores of the distributor and hence bed expansion takes place. Values are noted down for both increase and decrease in the superficial velocity for both expansion of the bed and pressure drop respectively.

\section{Results And Discussion}

It was observed experimentally that mechanical agitator like stirrer helped break up the channeling and spouting in a bed of nanosized powders. Previous studies on micron-sized powder fluidization (Iwa- date and Horio, 1998; Pacek and Nienow, 1990; Zhou and Li,1999) and previous studies on nanoparticle fluidization (Cha- ouki et al., 1985; Morooka et al., 1988; Wang, Y., et al., 2002; Wang, Z., et al., 1998) have shown that formation of agglomerates and their fluidization could be achieved at gas velocities well in excess of the minimum fluidization velocity, based on the diameter of the nanoparticles. Considerably smaller gas velocities (but still much larger than that based on the primary nanoparticle size) were adequate in our experiments, given that stirrer provided sufficient energy to the system to overcome interparticle forces and form stable agglomerates.

\section{- Bed expansion}

For APF nanoparticles, it is observed that the bed fluidizes and expands very uniformly without bubbles with alarge expansion ratio up to $200 \%$, thebed expansion increases with increasing gas velocity, and the agglomerates distribute uniformly within thebed. Asshown in Figure, for ABF nanoparticles, the bed expands very little with increasing gas velocity (lessthan 50\%), andlargebubbles riseupveryquickly through thebed, andthe agglomerates distribute non-uniformly within thebed,thesmaller agglomerates appearto besmoothly fluidized intheupperpartofthebed,while thelargeragglomerates couldbe foundmoving slowlyatthebottom.

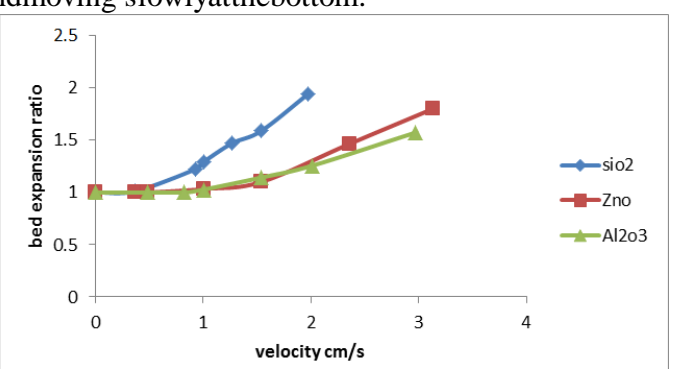

Figure 2 Effect of velocity on bed expansion for different nanoparticle without stirrer 


\section{- Effect of stirrer on bed expansion}

Thebedexpansionratioisobservedtobeincreaseswiththeincreasedspeedofthe

thismaybeduetothebedmaterialsof column movesvigorouslyandtheairbubblesarebreakduetocollisionamongparticles. As aresultthe bedexpansiondecreaseswithincreaseofspeedofstirrer.

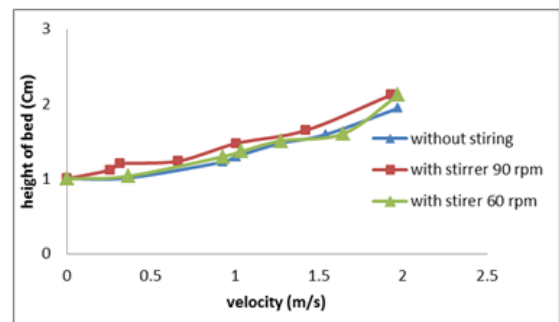

Figure 3 Effect of stirrer velocity $60 \mathrm{rpm}, 90 \mathrm{rpm}$ and without stirrer on bed expansion, initial bed height $4.25 \mathrm{~cm}, \mathrm{SiO2}$ nanoparticle size of $15 \mathrm{~nm}$

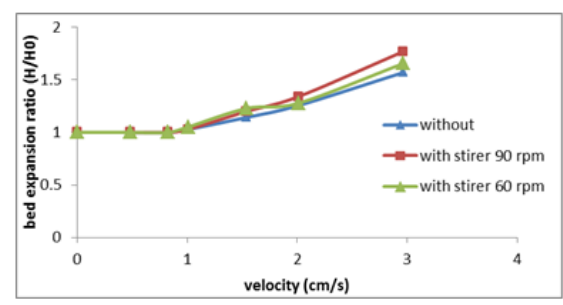

Figure 4 Effect of stirrer velocity 60rpm, $90 \mathrm{rpm}$ and without stirrer on bed expansion, initial bed height $1.75 \mathrm{~cm}$ ,Al2O3 nanoparticle size of $25 \mathrm{~nm}$

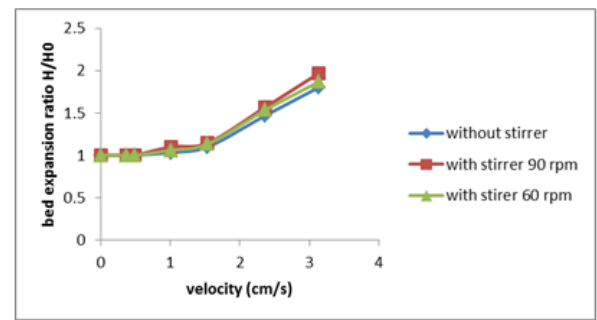

Figure 5 Effect of stirrer velocity 60rpm, $90 \mathrm{rpm}$ and without stirrer on bed expansion ,initial bed height $1.5 \mathrm{~cm}$ ,ZnO nanoparticle size of $30 \mathrm{~nm}$

- Effect of stirrer on bed voidage

it is observed that thebedvoidage of different nanoparticles size isincreasedwiththeincreaseinsuperficial gas velocity $\left(U_{0}\right)$ forthefluidizedbed an so also increase with speedofthestirrerand thusindicatingthe differentfluidizationregimesin the column. Highly porous particles show particulate and bubbling fluidization behavior. The graph of voidage vs. superficial is beneficial in this aspect determination.

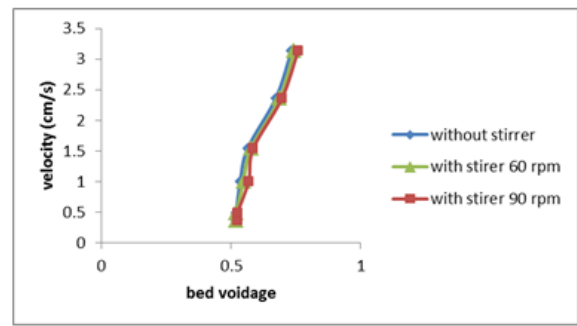

Figure 6 Effect of stirrer velocity $60 \mathrm{rpm}, 90 \mathrm{rpm}$ and without stirrer on bed voidage, initial bed height $1.5 \mathrm{~cm}, \mathrm{ZNO}$ nanoparticle size of $30 \mathrm{~nm}$

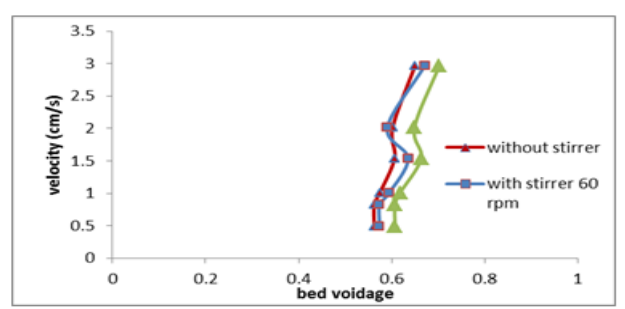

Figure 7 Effect of stirrer velocity 60rpm , $90 \mathrm{rpm}$ and without stirrer on bed voidage ,initial bed height $1.75 \mathrm{~cm}, \mathrm{Al} 2 \mathrm{O}$ nano particle size $25 \mathrm{~nm}$ 


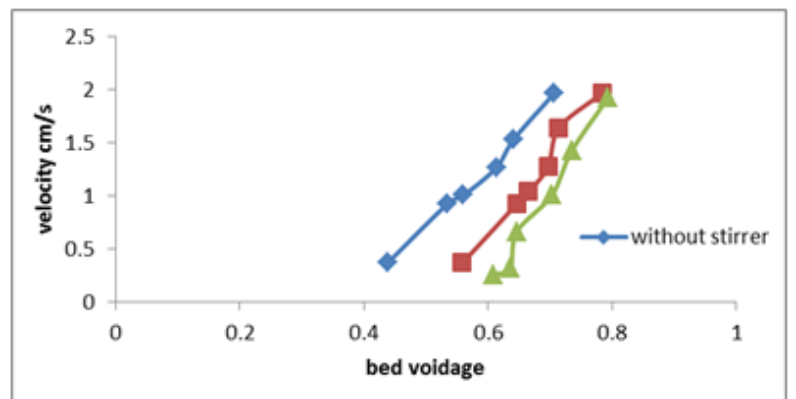

Figure 8 Effect of stirrer velocity 60rpm, $90 \mathrm{rpm}$ and without stirrer on bed voidage, initial bed height $4.25 \mathrm{~cm}, \mathrm{SIO2}$ nanoparticle size of $15 \mathrm{~nm}$

\section{- $\quad$ Agglomerate Sizes for APF nanoparticles (Modified Richadson-Zaki Equation)}

typical plot of Upt1/n Vs $\mathrm{H} 0 / \mathrm{H}$ for the APF nanoparticles ( $\mathrm{SiO} 2$ nano particle,primary size $15 \mathrm{~nm}$ ), with $\mathrm{n}=5$ is shown in ; all of the data points fall within the zone between the inner and outer solid lines. The figure also shows that all of the straight lines intersect the y-axis fairly close to each other, indicating that the terminal velocities for this nanoparticles are roughly of the same order of magnitude.

It is observed that with the assist of stirrer by increasing in the speed of stirrer initial bed voidage increased and terminal velocity and agglomerate diameter decreased and found that there are good match between experimental values for bed voidage and calculated values which is shown in table 1

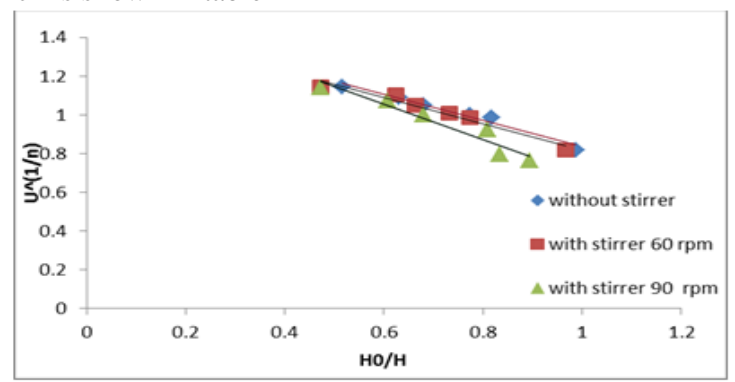

Figure $9 U^{\wedge}(1 / n)$ vs. H0/H Richardson-Zaki exponent $n=5$ on different stirrer velocity

Table 1 calculated agglomerate size and initial bed voidage of SIO2 APF nano particle (n=5)

\begin{tabular}{|c|c|c|c|}
\hline Material $(\mathrm{SiO2})$ & $\mathrm{U}_{\mathrm{pt}}(\mathrm{cm} / \mathrm{s})$ & $\xi_{0}$ & $\mathrm{D}_{\mathbf{a}}(\mu \mathrm{m})$ \\
\hline Without stirrer & 10.55146 & $\mathbf{0 . 4 3 1 0 2 4}$ & 344 \\
\hline Stirrer 60 rpm & 7.87109 & 0.542754 & 297 \\
\hline Stirrer 90 rpm & 7.568471 & 0.562754 & 292 \\
\hline
\end{tabular}

\section{Conclusion}

The results showed that smoother fluidization was observed with increasing agitation speed, because the agglomeration and channeling were reduced by the mechanical agitation.

- Itwas found for APF nano particle that with the assist of stirrer, by increasing in the speed of stirrer initial bed voidage increased and terminal velocity and agglomerate diameter decreased.

- Thepreliminarystudyhasshownthatfluidizationof nanoparticlecanbeeasily and smoothlyfluidizedwiththeassistanceof externalforce.Thusitcanbeconcludedthatapplying externalforcei.e.centrifugalforceimproves thebeddynamicstoagreatextentthereby minimizingtheenergyconsumption. Thereforeanexternalarrangementforcreatingsomeforce onoutsideofthecolumnwillbebestoptiontoimprovethebeddynamicsaswellaseconomyof theprocess.

- Thebedexpansionratioand bed voidageforthefluidizedbedareobserved toincrease with theincreasedspeedof thestirrer(N)astherotationofthestirrerpreventsthebubbleformation, reducing channeling and agglomeration.

\section{References}

[1]. Jaber Shabanian, Rouzbeh Jafari, Jamal Chaouki, Fluidization of Ultrafine Powders, Praise Worthy Prize (2012) 2035-1755

[2]. Wang Z., Kwauk M., Li H.Fluidization of fine particle, Chemical engg. Science, 53(1998) pp.377-395

[3]. Geldart, D., "Types of Gas Fluidization," Powder Technol., 7, 285 (1973)

[4]. Xu C., X-J.Effect of gas type on fine particle fluidization, China Particuology 4(2006) Pp. 3-4, 114-121

[5]. Chirone R., Russo S.Sound assisted fluidized bed combustion of fine particle, Combustion Science and technology, 153, pp.83-93

[6]. Rajesh.N, Yu Qun .Enhanced fluidization of nano particle in an oscillating Dave magnetic field, AICHE Journal, 51(2005):pp.19711977

[7]. Liu Guangliang, Zhu Chao, Dave Rajesh.N. Sound assisted fluidization of ano particle, Powder Technology 141(2004) 119-123

[8]. C. Zhu, Q. Yu, R. N. Dave, R. Pfeffer, Gas fluidization characteristics of nanoparticle agglomerates, AIChE J. 51 (2005)426-439.

[9]. D. Kunii, O. Levenspiel, Fluidization Engineering (Butterworth-Heinemann, 1991). 